\title{
A complication of subclavian venous catheterization: extravascular kinking, knotting, and entrapment of the guidewire
}

\section{-A case report-}

\author{
Jae Jun Lee ${ }^{1}$, Joo Sung Kim, Woon Seob Jeong ${ }^{2}$, Do Young Kim², Sung Mi Hwang ${ }^{1}$, and So Young Lim \\ Departments of ${ }^{1}$ Anesthesiology and Pain Medicine, ${ }^{2}$ Orthopedic Surgery, School of Medicine, Hallym University, Chuncheon, Korea
}

Various complications of central venous catheterization have been reported, some of which are well-known, while others are described as a sporadic events. We experienced a case of left subclavian venous catheterization complicated by extravascular knotting, kinking, and entrapment of the guidewire and the guidewire was removed surgically. Although minimal resistance was encountered during guidewire insertion, the guidewire was advanced approximately $30 \mathrm{~cm}$. Physicians should be aware of these rare potential complications when a guidewire is advanced if any resistance is encountered. (Korean J Anesthesiol 2010; 58: 296-298)

Key Words: Central venous catheterization, Complications.

Central venous catheterization (CVC) is commonly used for diagnosis and treatment, especially in critically ill patients, but CVC can have many severe complications, even for experienced physicians [1]. Mechanical complications of CVC occur in 5\% to $19 \%$ of patients $[2,3]$ and complications associated with the guidewire include failure to pass, loss in the vessel, kinking, and breakage. There has been no report of kinking, knotting, or entrapment of a guidewire, although intravascular catheter looping and knotting are well-known complications of CVC $[4,5]$. We present a case of extravascular kinking, knotting, and entrapment of a J-tipped flexible guidewire and successful surgical removal of the wire.

\section{Case Report}

An 88-year-old woman with a history of hypertension, atrial fibrillation, and unstable angina suddenly developed left-side motor weakness and right-side eyeball deviation. She was admitted to a district hospital for evaluation and management. Her diagnosis was right middle cerebral artery (MCA) infarction and she was given tissue plasminogen activator (tPA). Seven days later, she suddenly developed dyspnea and unstable

Received: June 24, 2009. Revised: July 17, 2009. Accepted: September 4, 2009.

Corresponding author: Woon Seob Jeong, M.D., Department of Orthopedic Surgery, School of Medicine, Hallym University, Gyo-dong, Chuncheon 200-704, Korea. Tel: 82-33-240-5780, Fax: 82-33-252-0941, E-mail: gogjus@hallym.or.kr

(c) This is an open-access article distributed under the terms of the Creative Commons Attribution Non-Commercial License (http:// creativecommons.org/licenses/by-nc/3.0/), which permits unrestricted non-commercial use, distribution, and reproduction in any medium, provided the original work is properly cited. 
vital signs: blood pressure $80 / 40 \mathrm{mmHg}$, heart rate 150 beats/ min, respiration 44 breaths $/ \mathrm{min}$, body temperature $39.1^{\circ} \mathrm{C}$, and $\mathrm{O}_{2}$ saturation $94 \%$. Under the impression of septic shock, she was intubated and left subclavian venous catheterization was attempted because of the need for continued fluid management. Lidocaine was injected at the entry site in the left infrasubclavian area. Then, the left subclavian vein was identified after several attempts using an infraclavicular approach. A Seldinger-type central venous catheter set was used (Two-Lumen Central Venous Catheterization Set with Blue FlexTip, ARROW, USA). The guidewire was passed through the introducer needle, which had been placed with venous return. Minimal resistance was encountered during guidewire insertion, and the guidewire was advanced approximately 30 $\mathrm{cm}$. At that point, the guidewire could be advanced no further and subsequent attempts to withdraw the guidewire were failed despite moderate force. A chest X-ray showed that the guidewire was knotted and kinked and the patient was transferred to our hospital for surgical exploration.

After admission to our hospital, a portable chest X-ray and subclavian venogram were performed for further evaluation. The X-ray revealed that the guidewire was knotted and kinked in the area of the middle third of the left clavicle and that part of it had folded back on itself completely and entered the mediastinum (Fig. 1). The subclavian venogram revealed that the guidewire had not perforated the subclavian vein and was knotted, kinked, and extended extravascularly. The subclavian vein was intact (Fig. 2).

Following discussion with orthopedic and chest surgeons, surgical exploration under general anesthesia was decided. In the operating room, the patient's initial vital signs were blood

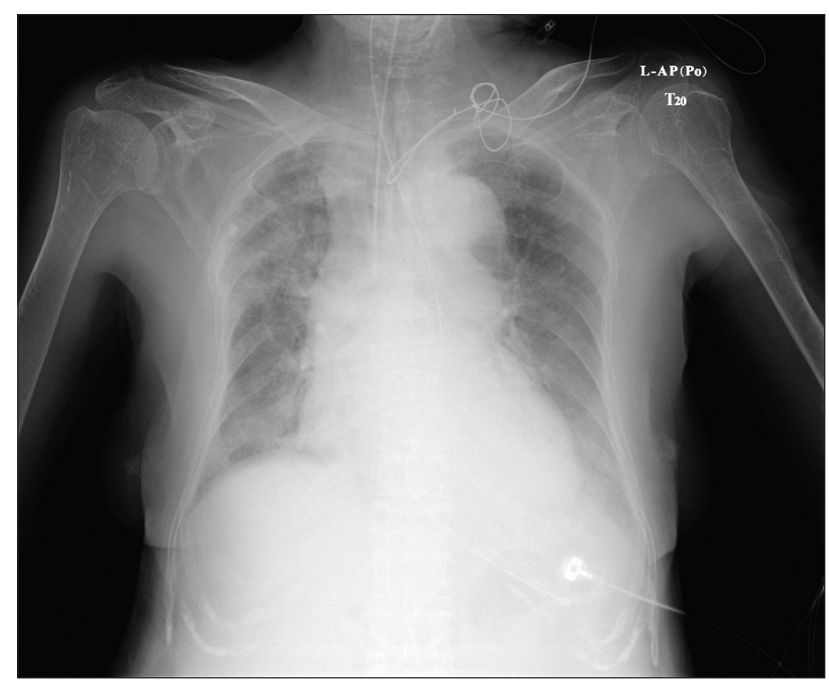

Fig. 1. Chest X-ray showing the knotted, kinked, and entrapped guidewire. pressure 100/50 $\mathrm{mmHg}$, heart rate 134 beats/min, respiration 25 breaths/min, and $\mathrm{O}_{2}$ saturation $96 \%$. Anesthesia was induced with $8 \mathrm{mg}$ of etomidate and $40 \mathrm{mg}$ of rocuronium. Although noninvasive blood pressure (NIBP) cuff had been applied on the right arm, an arterial catheter was placed in the left radial artery to continuously check the blood pressure and to promptly detect and monitor the blood pressure changes in case of the damage to the left subclavian artery during operation. And also a femoral venous catheter was applied at the left femoral vein. A 7-cm incision was made just medial to the entry point of the guidewire, parallel to Langer's line. Careful dissection revealed the guidewire just beneath the subclavian vein. The tangled part of the wire was kinked between the sternocleidomastoid muscles and the vessels were not damaged. We cut the wire to untangle it, and pulled out the wire that had entered the mediastinum. The entire guidewire was removed successfully, as confirmed under an image intensifier in the operating field. Postoperatively, the patient was transferred to the surgical intensive care unit, from which she was discharged to a ward the next day without further complications.

\section{Discussion}

We experienced a case of left subclavian venous catheterization complicated by extravascular knotting, kinking, and entrapment of the guidewire. While some studies have reported on intravascular knotting or looping of guidewires [6-9], a few cases of extravascular knotting, kinking, and entrapment of guidewires have been reported internationally and we believe that our case is the first domestic report. Wang and Einarsson [10] experienced a similar case, but on surgical cutdown, the wire

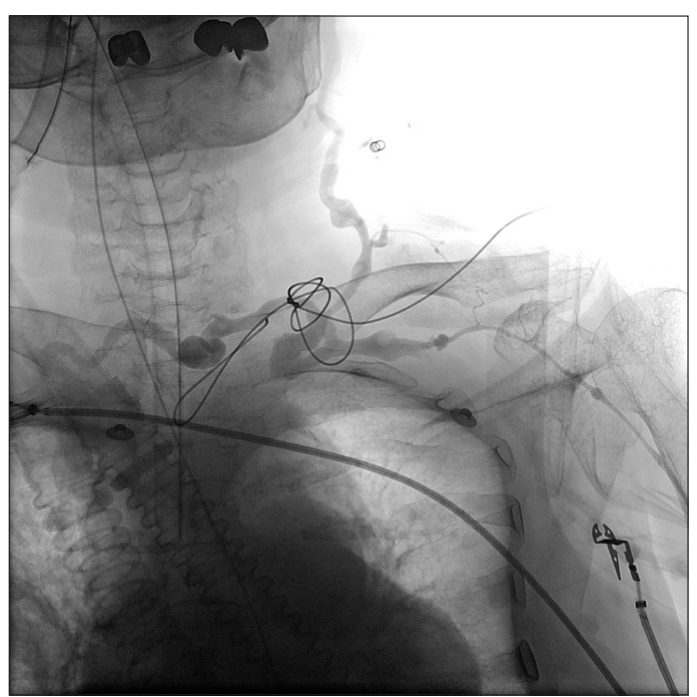

Fig. 2. Subclavian venogram showing the intact left subclavian vein. 
was discovered to perforate the proximal subclavian vein and knotted behind the clavicle. Their patient had a history of previous subclavian catheterization thus they postulated that damage to the vein from the previous catheterizations predisposed the vessel to perforation by the guidewire. In another similar case, surgical cutdown revealed that the wire had passed through a nearly occluded subclavian vein and into the anterior scalene muscle, where fibrous tissue redirected the wire upward and into a loop [11]. That patient also had a history of five previous subclavian catheterizations.

In our case, the patient had no history of subclavian catheterization and surgical exploration which means that the vessels were not perforated or damaged. The guidewire was located just beneath the subclavian vein and the tangled part of the wire was kinked between the sternocleidomastoid muscles. Furthermore, the first $12 \mathrm{~cm}$ of the guidewire from the J-tip was doubled back on itself and extended $6 \mathrm{~cm}$ into the mediastinum; no damage occurred to the J-tip.

From these facts, we inferred that the guidewire was initially inserted extravascularly, although the introducer needle had been placed with venous return. The intact subclavian vein and venogram findings supported this supposition. When the J-tip guidewire was inserted into the extravascular connective tissue area, it had folded into a U-shape, which then folded completely upon itself into an I-shape. Finally, this I-shaped wire passed through the connective tissues between the vessel and muscle into the mediastinum, while the rest of the wire, which was under continuous force, became kinked, knotted, and entrapped in the middle clavicular area. This likely resulted from the connective tissue around the subclavian vein and attachment of the vein to the surrounding structures [12]. The loose connective tissues of old age may also have been a contributing factor. Anatomically, the path of the subclavian vein is not straight, as it loops over the first rib to descend into the superior mediastinum. Furthermore, a bottleneck exists between the clavicle and first rib, which can impede the anterograde threading of the wire and might contribute to looping and knotting [6].

In our case, minimal resistance was encountered during guidewire insertion. Consequently, we emphasize that a guidewire should not be advanced if any resistance is encountered, as originally pointed out by Seldinger [13]. Guidewires are not very rigid structures and if any force is applied they are likely to kink, moreover, further application of force after kinking might result in knot formation [6]. If we pull out the knotted guidewire back, the guidewire might be entrapped. To avoid these complications, if there is any resistance, guidewire should not be advanced and resistance encountered during insertion of the guidewire, it is necessary to check the chest $\mathrm{x}$-ray to determine the position of the guidewire instead of pulling it back. Our case shows the consequences of inserting a guidewire despite of minimal resistance and pulling it backward when the guidewire is not advancing.

In conclusion, CVC is commonly practiced by anesthesiologists, surgeons, and emergency room physicians. We present a rare complication of subclavian venous catheterization. Initially, the guidewire was passed through extravascular connective tissue and then the wire became knotted, kinked, and entrapped extravascularly. Physicians should be aware of these rare potential complications when a guidewire is advanced if any resistance is encountered.

\section{References}

1. Boon JM, van Schoor AN, Abrahams PH, Meiring JH, Welch T, Shanahan D. Central venous catheterization-an anatomical review of a clinical skill-part 1: subclavian vein via the infraclavicular approach. Clin Anat 2007; 20: 602-11.

2. Merrer J, De Jonghe B, Golliot F, Lefrant JY, Raffy B, Barre E, et al. French Catheter Study Group in Intensive Care. Complications of femoral and subclavian venous catheterization in critically ill patients: a randomized controlled trial. JAMA 2001; 286: 700-7.

3. Mansfield PF, Hohn DC, Fornage BD, Gregurich MA, Ota D. Complications and failures of subclavian-vein catheterization. $\mathrm{N}$ Engl J Med 1994; 331: 1735-8.

4. Malatinsky J, Kadlic T, Majek M, Samei M. Misplacement and loop formation of central venous catheters. Acta Anaesthesiol Scand 1976; 20: 237-47.

5. Nicholas F, Fenig J, Richter RM. Knotting of subclavian central venous catheter. JAMA 1970; 214: 373-4.

6. Khan KZ, Graham D, Ermenyi A, Pillay WR. Case report: managing a knotted Seldinger wire in the subclavian vein during central venous cannulation. Can J Anesth 2007; 54: 375-9.

7. Leigh TR, Baker LR, Cattell WR. Internal knotting of a guidewire: a complication of subclavian vein cannulation. Nephron 1987; 46: 210.

8. Koundouris C, Tornaris G, Charopoulos I, Vounasis A, Solomou G. Subclavian vein flexible guidewire knotting. A potential serious complication in hemodialysis patients. Chirurgia (Bucur) 2004; 99 : $61-4$.

9. Kim SY, Jang YH. J-guide wire knotting during the central venous catheterization. Korean J Anesthesiol 2003; 44: 725-8.

10. Wang LP, Einarsson E. A complication of subclavian vein catheterisation. Extravascular knotting of a guidewire. Acta Anaesthesiol Scand 1987; 31: 187-8.

11. Kjeldsen L. Transvenous misplacement and loop formation of spring guide wire. Anaesthesia 1987; 42: 216-7.

12. Mothers LH, Smith DW, Frankel L. Anatomic considerations in placement of central venous catheters. Clin Anat 1992; 5: 89-106.

13. Seldinger SI. Catheter replacement of the needle in percutaneous arteriography: a new technique. Acta Radiol 1953; 39: 368-76. 